\title{
Dry eye disease: when to treat and when to refer
}

\section{Quan Findlay}

PhD candidate, University of Melbourne

\section{Kate Reid}

Senior staff specialist,

Department of

Ophthalmology, Canberra

Hospital

Clinical senior lecturer,

Australian National

University, Canberra

\section{Keywords}

aqueous deficiency dry eye, evaporative dry eye, meibomian gland dysfunction

Aust Prescr 2018;41:160-3

https://doi.org/10.18773/ austprescr.2018.048

\section{SUMMARY}

Dry eye disease affects one in five adults, and can significantly impair quality of life. Most patients have mild disease.

This condition is multifactorial, with an inflammatory component which can markedly worsen the impact on the ocular surface. Meibomian gland dysfunction is extremely common in dry eye disease, and contributes to the inflammatory process.

Management of mild disease includes identifying and removing precipitants, and symptomatic treatment with artificial tear supplements.

More advanced disease requires management of underlying ophthalmic and systemic conditions, as well as more aggressive therapies to protect the ocular surface.

\section{Introduction}

Dry eye disease, or keratoconjunctivitis sicca, is highly prevalent, and can have significant adverse effects on quality of life. It is 'a multifactorial disease of the ocular surface characterized by a loss of homeostasis of the tear film, and accompanied by ocular symptoms in which tear film instability and hyperosmolarity, ocular surface inflammation and damage, and neurosensory abnormalities play aetiological roles'.

As a leading cause of patient visits to both optometrists and ophthalmologists, it is a substantial burden on the healthcare system. While most patients have mild disease and need only simple treatment, complex interventions in severe disease aim to prevent progression to corneal ulceration and conjunctival scarring. It should be noted that dry eye disease is worsened by contact lens wear and refractive laser surgery.

\section{Dry eye disease subtypes}

There are two main subtypes of dry eye disease aqueous deficiency and evaporative. These may co-exist. ${ }^{2}$

\section{Aqueous deficiency}

Aqueous deficiency occurs because of reduced aqueous production from the lacrimal glands. It accounts for only a tenth of dry eye disease. Aqueous deficiency can be further separated into Sjögren's syndrome-related and non-Sjögren's syndrome-related.

\section{Evaporative}

Evaporative dry eye is due to a deficient tear film lipid layer, which increases tear evaporation. It is caused by meibomian gland dysfunction, which occurs in over $85 \%$ of dry eye disease. Blepharitis, or lid margin inflammation, is both a cause and an effect of meibomian gland dysfunction. The differential diagnosis of blepharitis includes ocular rosacea and atopy, seborrhoeic dermatitis, staphylococcal infection and Demodex mite infestation. Tear deficiency is thought to alter resistance to infection, so dry eye disease is both a cause and an effect of blepharitis.

\section{Risk factors}

Multiple factors contribute to the development of dry eye disease (Box 1). Female gender is the most consistently identified risk factor, with the prevalence in women being almost double that in men. Advancing age is also a major risk factor, possibly due to decreased androgen levels, since androgen up-regulates meibomian gland function in animal models. While oestrogen is thought to down-regulate it, there is uncertainty as to whether hormone replacement therapy exacerbates or improves dry eye disease. ${ }^{3}$

\section{Diagnosis and assessment}

Accurately diagnosing dry eye disease and determining its severity is confounded by the variability in clinical presentation. Patients often report non-specific symptoms, such as visual disturbance, photophobia and ocular discomfort, including foreign body sensation, grittiness and burning. 
Paradoxically there may be excessive wateriness, as discomfort triggers reflex tearing. The severity of symptoms does not correlate well with the severity of signs seen at the slit lamp, especially if there is a low pain threshold (symptoms exceed signs), or if there is reduced corneal sensation (signs exceed symptoms).

All of these symptoms may be present in other unrelated eye conditions such as ocular allergy, corneal erosion and foreign body. Differential diagnoses must be considered when symptoms are severe or unilateral. Box 2 outlines when referral for specialist assessment is needed. ${ }^{4}$

Dry eye questionnaires such as DEQ-5 or OSDI have been developed and validated for screening and/or measuring severity and response to treatment. However, they are not in common use. Clinical assessment and various tests are required to diagnose dry eye disease.

\section{Ocular surface staining}

In dry eye disease, there is loss of the protective glycocalyx barrier, due to increased shedding of the corneal and conjunctival epithelial cells. ${ }^{5}$ The new underlying cells are able to absorb vital dyes, with the degree of pathological staining matching disease severity. Areas of epithelial cell loss are readily seen using sodium fluorescein drops under cobalt blue light at the slit lamp.

\section{Tear film break-up time}

Tear film break-up time is reduced in dry eye disease. Again using sodium fluorescein drops under cobalt blue light at the slit lamp, the time it takes for the first dark spot to appear in the fluorescein-stained tear film since the last complete blink is measured. A tear break-up time less than 10 seconds indicates tear film instability, and a measurement less than five seconds diagnoses dry eye disease.

\section{Tear lake}

The tear meniscus seen at the inferior lid margin is reduced in dry eye disease. Less than $0.2 \mathrm{~mm}$ is diagnostic.

\section{Blepharitis}

Debris is seen on the lashes and there may be reddening, telangiectasia and thickening of the lid margins.

\section{Meibomian gland assessment}

Objective meibomian gland assessment is important for diagnosing evaporative dry eye disease. Healthy meibomian glands should discharge a transparent liquid oil under gentle pressure to the lid margin, whereas thick or discoloured meibum indicates gland dysfunction. 'Pouting' of the gland orifices at the lid margin may be seen due to retained meibum.

\section{Box 1 Causes and risk factors for dry eye disease}

- Female gender and advancing age, possibly hormone replacement therapy

- Blepharitis/meibomian gland disease - rosacea, seborrhoeic dermatitis, staphylococcal infection, Demodex mite infestation

- Lagophthalmos - facial nerve palsy, proptosis, vertical lid shortening

- Decreased blinking - prolonged computer use or other visual task, Parkinson's disease

- Ocular autoimmune disease - atopy, cicatricial pemphigoid

- Systemic autoimmune disease - Sjögren's syndrome, lupus, scleroderma, chronic graftversus-host disease, rheumatoid arthritis

- Other medical causes - vitamin A deficiency, hepatitis C, thyroid disorders

- Antihypertensives, antihistamines and antidepressants

- Exogenous factors - radiation therapy, chemical injuries

- Low-humidity environments e.g. air conditioning or heating

- Low intake of omega-3 fatty acids

\section{Box 2 NICE guidelines for referral to an optometrist or ophthalmologist}

- Patients with moderate-severe eye pain, photophobia, marked redness in one eye or reduced visual acuity (same day referral)

- Deteriorating vision

- Ulcers or signs of corneal damage

- Persisting or worsening symptoms despite appropriate treatment for four weeks

- Associated disease requiring specialist treatment e.g. Sjögren's syndrome, eyelid deformities

Source: reference 4

\section{Schirmer's test}

Schirmer's test is used to diagnose aqueous deficiency dry eye disease. It is more invasive than the tests above, as a Schirmer strip composed of filter paper is placed into the lower fornix for five minutes. Anaesthetic drops are instilled first, to prevent reflex tearing due to irritation by the paper. A measurement less than $5 \mathrm{~mm}$ is consistent with low aqueous tear production.

\section{Other tests}

Tears can also be analysed for hyperosmolarity, and for the cytokine MMP-9 as a marker of inflammation and dryness. ${ }^{6}$

\section{Management}

Treatment of dry eye disease aims to relieve symptoms, and to reduce any risk of ocular surface damage. Tear film homeostasis should be restored as much as possible.

\section{Mild disease}

Box 3 provides a summary of first-line treatments for mild dry eye disease. Many of the treatments for mild symptoms are available from the pharmacy (Table). 


\section{Box 3 First-line treatments for mild dry eye disease}

- Apply ocular lubricants - drop, gel or ointment depending on severity of symptoms, preferably unpreserved. Consider adding a lipid layer stabiliser.

- Treat blepharitis - lid wipes, rosacea management, eradication of infection e.g. oneweek course of chloramphenicol ointment to lid margins.

- Optimise meibomian gland function - warm compresses, warming eye masks.

- Modify the environment to decrease evaporation of tears - increase air humidity, reduce computer use, increase frequency of breaks for eye rest, 'conscious blinking',

- Review drugs that may exacerbate eye symptoms e.g. antihistamines, beta blockers, tricyclic antidepressants, selective serotonin reuptake inhibitors, isotretinoin, eye drops with preservatives.

\section{Table Examples of pharmacy treatments for dry eye disease}

\begin{tabular}{lll}
\hline Main lubricant & Presentation & Preserved \\
\hline 'Aqueous' tear supplements & & \\
\hline Carmellose sodium (Cellufresh, Celluvisc) & Single-use vial & No \\
\hline $\begin{array}{l}\text { Polyethylene glycol or propylene glycol } \\
\text { (Systane drops or gel drops) }\end{array}$ & Single-use vial & No \\
\hline $\begin{array}{lll}\text { Sodium hyaluronate (Hylo-Fresh, Hylo-Forte) } \\
\text { Carbomer (Poly Gel) }\end{array}$ & Multi-dose bottle & Yes \\
\hline $\begin{array}{l}\text { Carboxymethylcellulose sodium, hypromellose } \\
\text { (Genteal gel) }\end{array}$ & Single-use vial & No \\
\hline
\end{tabular}

\section{'Lipid’ tear supplements}

\begin{tabular}{llc}
\hline $\begin{array}{l}\text { Propylene glycol, emulsified mineral oil } \\
\text { (Systane Balance) }\end{array}$ & Multi-dose bottle & Yes \\
\hline Soya lecithin (Optrex ActiMist) & $\begin{array}{l}\text { Multi-dose spray } \\
\text { (closed lids) }\end{array}$ & Yes \\
Perfluorohexyloctane (NovaTears) & Multi-dose bottle & No \\
\hline
\end{tabular}

\section{Lid cleansers}

$\begin{array}{lll}\text { Foam solution containing plant oils (Sterilid) } & \text { Pump bottle } & \text { No } \\ \text { Lid wipes (Systane) } & \text { Wipes } & \text { No }\end{array}$

\section{Lubricants}

Artificial tear drops to supplement the aqueous component of the tear film are the first-line therapy for patients with mild symptoms. For moderate symptoms, gels are used during the day. Lubricating ointment is only applied at night, as it causes blurring of vision. The regular use of artificial tears, gels and ointment increases tear film break-up time, and reduces signs of corneal damage a month's treatment produces improvement of around 25\%.7 An insert retained by the lower lid (Lacrisert) provides a slow-release alternative to conventional lubricants. Newer preparations seek to stabilise the lipid layer of the tear film, and can be used in conjunction with lubricants augmenting the aqueous layer.

\section{Preservatives}

Preservatives such as benzalkonium chloride are commonly found in eye drops, including artificial tears, corticosteroids, antibiotics and glaucoma medicines. These can cause irritation and exacerbate dry eye disease. However, because preservatives are diluted in the tear film, they remain suitable for patients with mild dry eye. In more severe disease, the dilution effect is attenuated due to reduced tear volume, so preservative-free eye drops are recommended.

\section{Meibomian gland dysfunction}

Every effort must be made to treat blepharitis and meibomian gland dysfunction. Strategies include using lid wipes or foam cleansers, doxycycline for ocular rosacea, warm compresses or eye masks, and expression of blocked glands.

Extrapolating from its use in facial rosacea, some optometrists and ophthalmologists now offer intense pulsed light therapy to improve meibomian gland function. Treatment is applied across the zygomatic arches, lower lids and bridge of the nose, while the patient is wearing opaque goggles. A variety of treatment mechanisms are proposed, ${ }^{8}$ but there are limited studies to date. A thermal/pulsation system (LipiFlow) provides an automated method of lid margin heating and massage, and is also aimed at improving meibomian gland function.

\section{Refractory disease}

Certain patient populations have more refractory disease and require more aggressive intervention to reduce the risk of permanent ocular surface injury. This includes patients with rheumatoid arthritis or Sjögren's syndrome, and those with cicatrising disease of the conjunctiva, such as severe atopy or ocular pemphigoid. Here the foundation of care is optimal treatment of the underlying systemic disease, with co-management of the patient by both an ophthalmologist and an immunologist or rheumatologist.

\section{Medical treatments}

Topical anti-inflammatory drugs are used by ophthalmologists for more severe cases. However, topical corticosteroids are sparingly prescribed, due to the risk of glaucoma, infection and keratolysis.

Immunomodulatory drugs with anti-inflammatory effects such as ciclosporin eye drops (0.05-0.1\%) have been shown to reduce symptoms and corneal surface damage. ${ }^{9}$ Tacrolimus eye drops (0.02-0.03\%) are a viable alternative for patients who are unable to use ciclosporin, or do not benefit from it. ${ }^{10}$ Testosterone eye drops (0.03\%) have shown promise in very limited settings," but like tacrolimus can only be obtained from a compounding chemist. 
Autologous serum eye drops, containing growth factors, vitamin A and fibronectin, are effective in severe dry eye disease. However preparation is laborious, and the procedure is only available in hospitals. ${ }^{12}$

\section{Surgical treatments}

Reduction of tear drainage by punctal occlusion, with dissolvable or permanent plugs, has been shown to provide symptomatic improvement, particularly in aqueous deficiency dry eye disease and when combined with other treatments. Permanent surgical closure is offered if clinical benefit is obtained from temporary plugs.

Severe lagophthalmos may need to be addressed with botulinum toxin-induced ptosis if temporary, or tarsorrhaphy if permanent.

\section{Referring appropriately}

GPs and pharmacists are well placed to recommend the interventions in Box 3 for mild disease. For more severe symptoms it is appropriate to refer a patient to an optometrist before an ophthalmologist.
An optometrist can perform a specialised eye examination, including a comprehensive dry eye disease evaluation, using equipment that is not normally available in general practice. The National Institute for Health and Care Excellence provides concise recommendations on when to refer patients (Box 2). ${ }^{4}$

\section{Conclusion}

Dry eye disease is common, and particularly prevalent in older women. Management of mild disease consists of tear supplements from the pharmacy as first-line treatment, and techniques to manage meibomian gland dysfunction. Patients with more severe symptoms or risk factors for ocular surface damage can be assessed by an optometrist, then referred to an ophthalmologist as needed for more advanced interventions. «

Conflict of interest: none declared

Acknowledgement: The authors would like to thank Dr Laura Downie for her assistance in editing this review.

\section{REFERENCES}

1. Craig JP, Nichols KK, Akpek EK, Caffery B, Dua HS, Joo CK et al. TFOS DEWS II definition and classification report. Ocul Surf 2017;15:276-83. https://doi.org/10.1016/ j.jtos.2017.05.008

2. Lemp MA, Crews LA, Bron AJ, Foulks GN, Sullivan BD. Distribution of aqueous-deficient and evaporative dry eye in a clinic-based patient cohort: a retrospective study. Cornea 2012;31:472-8. https://doi.org/10.1097/ICO.0b013e318225415a

3. Peck T, Olsakovsky L, Aggarwal S. Dry eye syndrome in menopause and perimenopausal age group. J Midlife Health 2017;8:51-4. https://doi.org/10.4103/jmh.JMH_41_17

4. National Institute for Health and Care Excellence. Clinical Knowledge Summaries: Dry eye syndrome. United Kingdom: NICE; 2012.

5. Bron AJ, Argüeso P, Irkec M, Bright FV. Clinical staining of the ocular surface: mechanisms and interpretations. Prog Retin Eye Res 2015;44:36-61. https://doi.org/10.1016/ j.preteyeres.2014.10.001

6. Messmer EM, von Lindenfels V, Garbe A, Kampik A. Matrix metalloproteinase 9 testing in dry eye disease using a commercially available point-of-care immunoassay. Ophthalmology 2016;123:2300-08. https://doi.org/10.1016/ j.ophtha.2016.07.028

7. Doughty MJ, Glavin S. Efficacy of different dry eye treatments with artificial tears or ocular lubricants: a systematic review. Ophthalmic Physiol Opt 2009;29:573-83. https://doi.org/10.1111/j.1475-1313.2009.00683.x
8. Dell SJ. Intense pulsed light for evaporative dry eye disease. Clin Ophthalmol 2017;11:1167-73. https://doi.org/10.2147/ OPTH.S139894

9. Sacchetti M, Mantelli F, Lambiase A, Mastropasqua A, Merlo D, Bonini S. Systematic review of randomised clinical trials on topical ciclosporin A for the treatment of dry eye disease. Br J Ophthalmol 2014;98:1016-22. https://doi.org/ 10.1136/bjophthalmol-2013-304072

10. Sanz-Marco E, Udaondo P García-Delpech S, Vazquez A, Diaz-Llopis M. Treatment of refractory dry eye associated with graft versus host disease with $0.03 \%$ tacrolimus eyedrops. J Ocul Pharmacol Ther 2013;29:776-83. https://doi.org/10.1089/jop.2012.0265

11. Dawson TL. Testosterone eye drops: a novel treatment for dry eye disease. Ophthalmol Times 2015 Nov 15. http://www.ophthalmologytimes.com/modern-medicinefeature-articles/testosterone-eye-drops-novel-treatmentdry-eye-disease [cited 2018 Sep 1]

12. Quinto GG, Campos M, Behrens A. Autologous serum for ocular surface diseases. Arq Bras Oftalmol 2008;71(6 Suppl):47-54. http://dx.doi.org/10.1590/S0004-27492008000700010

\section{FURTHER READING}

Dry eye redefined: TFOS DEWS II report [Internet]. Boston (MA): Tear Film \& Ocular Surface Society; 2017. www.tfosdewsreport.org/ index.php?lng=en [cited 2018 Sep 1] 